\title{
COMMENTARY
}

\section{Crystalloids versus colloids during acute normovolemic anemia: the quest continues...}

\author{
Karl Schebesta* and Oliver Kimberger \\ See related research by Pape et al., http://ccforum.com/content/16/2/R69
}

\begin{abstract}
The optimal kind of fluid for fluid resuscitation during acute, severe hemorrhage is still discussed controversially. Of particular interest in this context is the choice of colloids versus crystalloids and their effect on the critical hemoglobin level. In a previous issue of Critical Care, Pape and colleagues describe the effect of four different volume replacement options on the critical hemoglobin concentration, and show marked differences for the different treatments. Even though some important pathophysiological issues remain unsolved, the current manuscript adds interesting evidence to an ongoing quest.
\end{abstract}

Pape and colleagues discuss the effect of different volume replacements in a previous issue of Critical Care [1]. In light of the numerous risks associated with allogeneic blood transfusions, many efforts have been made towards blood-sparing management of the perioperative phase [2]. By inducing normovolemic, dilutional anemia, the amount of blood transfusions given throughout surgery can be reduced; however, an adequate oxygen supply/ demand relationship and resulting adequate tissue oxygenation are the limiting factors when applying this technique. When the hemoglobin concentration falls below the level where oxygen delivery does not meet the tissue oxygen demand anymore, a critical hemoglobin level $\left(\mathrm{Hb}_{\text {crit }}\right)$ has been reached and outcome is poor without intervention, that is, the administration of red blood cells [3]. Also during acute, accidental blood loss the $\mathrm{Hb}_{\text {crit }}$ can rapidly ensue. Recommendations and guidelines for treatment of acute, accidental hemorrhage have changed over the last years and continue to evolve.

*Correspondence: karl.schebesta@meduniwien.ac.at

Department of Anesthesia, General Intensive Care and Pain Management, Medical University of Vienna, Währinger Gürtel 18-20, A-1090 Vienna, Austria
Pape and colleagues add evidence for the ongoing quest for optimal fluid replacement strategies during acute hemorrhage and address the unresolved controversy of crystalloid versus colloid fluid replacement strategies with the help of an established porcine hemorrhage model [1]. In this particular porcine model Pape and colleagues simultaneously withdrew blood and replaced the volume with 'plasma-expansion-rate-adjusted' amounts of Ringer's solution, gelatine, 6\% hydroxyethyl starch (HES) $130 / 0.4$ or $6 \%$ HES $450 / 0.7$ and assessed the $\mathrm{Hb}_{\text {crit }}$ by repetitively measuring oxygen uptake $\left(\mathrm{VO}_{2}\right)$ in conjunction with a computed regression analysis to detect deterioration indicating critical hemoglobin values. With this technique the authors could show that the choice of fluid replacement impacts the onset and extent of the $\mathrm{Hb}_{\text {crit }}$. In conclusion, the authors propose tetrastarch preparations (for example, HES 130/0.4) as the 'most advantageous regarding maintenance of tissue oxygenation during progressive anemia' [1]. However, this present study undoubtedly has some limitations that suggest a more careful approach regarding the interpretation of its results.

Foremost, the induced blood loss was replaced with different solutions at different ratios, with the intention to account for the different plasma expansion effects. However, the stroke volume variation, as indicator for volume responsiveness, decreased more in all but the Ringer's solution group, while intrathoracic blood volume (ITBV) increased in the colloid groups. Consequently, normovolemia seems to have been maintained best in the Ringer's solution group, while hypervolemic anemia was induced in the other three groups. The authors addressed this fact by discussing volume responsiveness and the possibility of having infused Ringer's solution at higher rates of 4:1 or 5:1. Yet, as normovolemic anemia was the goal, the colloidal infusions could have been infused at a lower rate. As rheologic effects and augmentation of regional blood flow during fluid replacements play a major role in acute hemodilution, simply using a fixed blood/fluid exchange rate based on the theoretical plasma expansion effect to address individual fluid demand may not have been the optimal approach. Using 
a goal-directed fluid therapy instead could have been more useful and addressed the actual fluid demands of the individual animals during severe bleeding (for example, a difference in pulse pressure (dPP)-guided fluid regimen) [4].

Furthermore, even though the critical hemoglobin concentration is the absolute limiting factor of hemodilution, adverse effects could have probably been seen at earlier stages [5]. As the cardiac oxygen extraction cannot be augmented like in other tissues, oxygen delivery and cardiac index are the actual limiting factors of hemodilution. In mild hemodilution, coronary oxygen delivery is improved by a decreased plasma viscosity. Redistribution of blood flow from other organs to the heart accounts for the preservation of cardiac oxygenation during more pronounced hemodilution. During severe hemodilution, exhaustion of subendocardial flow reserves will cause local ischemia and is the main complication of further blood loss. As shown in the study by Pape and colleagues [1], cardiac index did significantly increase in all groups until the $\mathrm{Hb}_{\text {crit }}$ was reached in the Ringer's solution. Thereafter, only small changes could be found. However, when looking at the oxygen delivery, an even decrease can be found in all groups, indicating no beneficial effects of any of the solutions at the $\mathrm{Hb}_{\text {crit }}$ of the Ringer's solution. Finally, the oxygen extraction ratio significantly increased from baseline in all but the Ringer's solution group at their respective $\mathrm{Hb}_{\text {crit }}$. Therefore, as coronar blood flow and cardiac oxygenation were not assessed, the actual limits of hemodilution with the solutions used are still not entirely clear.

As the authors stated in their discussion, the animals were terminated after reaching $\mathrm{Hb}_{\text {crit }}$ and only short-term effects were assessed. Thus, the displayed results do not account for local tissue hypoxia and other effects that would have resulted from redistribution of the infused fluids into the extravascular space as these effects would have needed additional time to be detectable.

Some statistical issues might also be mentioned. Even though the authors assessed for normal distribution and thereafter used multiple testing, they did not correct their $P$-values or their results for this fact. Corrections were only performed within a parameter. Inference from the results could have been drawn more cautiously, considering the extensive multiple testing of all parameters.

\section{Conclusion}

Subsequent trials will be needed to pathophysiologically explain and validate the results of the present study. Effects on a microcirculatory level of the different fluids at a $\mathrm{Hb}_{\text {crit }}$ need to be assessed in more detail and, as mentioned, implementation of a goal-directed regimen might be suggested. In summary, the authors are to be congratulated for adding evidence to the long-running debate on what type of fluid is best to use in hemorrhage.

\section{Abbreviations}

$\mathrm{Hb}_{\text {crit' }^{\prime}}$ critical hemoglobin concentration; HES, hydroxyethyl starch.

\section{Competing interests}

Neither of the contributing authors is biased by competing interests, including financial interests related to patents and/or share holdings in corporations involved in the development and/or marketing of the medication evaluated.

Published: 19 June 2012

\section{References}

1. Pape A, Kutschker S, Kertscho H, Stein P, Horn O, Lossen M, Zwissler B, Habler $\mathrm{O}$ : The choice of the intravenous fluid influences the tolerance of acute normovolemic anemia in anesthetized domestic pigs. Crit Care 2012, 16:R69.

2. Shander A, Rijhwani TS: Acute normovolemic hemodilution. Transfusion 2004, 44(12 Suppl):26S-34S.

3. Meier J, Kemming Gl, Kisch-Wedel H, Wolkhammer S, Habler OP: Hyperoxic ventilation reduces 6-hour mortality at the critical hemoglobin concentration. Anesthesiology 2004, 100:70-76.

4. Pestel GJ, Fukui K, Kimberger O, Hager H, Kurz A, Hiltebrand LB: Hemodynamic parameters change earlier than tissue oxygen tension in hemorrhage. J Surg Res 2010, 160:288-293.

5. Spahn DR, Leone BJ, Reves JG, Pasch T: Cardiovascular and coronary physiology of acute isovolemic hemodilution: a review of nonoxygencarrying and oxygen-carrying solutions. Anesth Analg 1994, 78:1000-1021.

doi:10.1186/cc11364

Cite this article as: Schebesta K, Kimberger O: Crystalloids versus colloids during acute normovolemic anemia: the quest continues.... Critical Care 2012, 16:131 